\title{
System accuracy evaluation of the systems for self- monitoring of blood glucose GL50 evo and GL 44 following DIN EN ISO 15197:2015: A comparison of accuracy in glucose concentration ranges $<100 \mathrm{mg} / \mathrm{dL}$ and $\geq 100 \mathrm{mg} / \mathrm{dL}$
}

\author{
Eckhard Salzsieder*, Sabine Berg, Anselm Puchert and Ernst-Joachim Freyse \\ Institute of Diabetes, Gerhardt Katsch, Karlsburg, Germany
}

\begin{abstract}
Ongoing standardized verification of the accuracy of blood glucose meters systems for self-monitoring post-launch is important clinically and helps confirm appropriate continues performance of self-monitoring blood glucose (SMBG) systems. In addition, publication of such studies is increasingly becoming a component of evidence-based purchase decision making. ISO 15197:2015,2 for which mandatory compliance is recommended for SMBG systems by 2015,3 has tighter accuracy requirements than ISO 15197:2003,4 and outlines current minimum accuracy standards necessary in Europe for CE marking.
\end{abstract}

\section{Introduction}

In the present study, a post marketing evaluation of the CE-marked GL50 evo and GL44 systems were performed in accordance with ISO 15197:2015 protocols and requirements. The GL50 evo and GL44 systems were supplied in Germany from the Beurer GmbH, Germany. A declaration of conformity from the manufacturer of the two measuring systems was available before the start of the study, so that only the GL50 evo was used in the tests, but the results documented the quality of both systems. Two GL50 evo systems (serial number: GL55 T1 and GL55 T2) and strips from 3 different lots (A 10/1, A 10/3, A 10/4) with expiry dates March 2017 respectively) were supplied by the manufacturer. The study was conducted from April 21 to May 05, 2015, at the Institute of Diabetes "Gerhardt Katsch," Karlsburg, Germany. Ethical approval for the study was obtained from the Ethics Committee of the University of Greifswald in July 2014 [1-3].

Ear lobe capillary blood samples were taken from 118 subjects for duplicate glucose determination using the GL50 evo and the glucose oxidase based YSI2300 STAT PLUS (YSI Incorporated, Yellow Springs, Ohio, USA) plasma glucose reference method. Trueness and precision of the comparison assay were verified using a range of YSI bioanalytical standards and controls. The prescribed limits for the hematocrit values, to be between $20 \%$ and $60 \%$, were reached by the patient samples and after examination of glucose concentration ranges using the YSI, 100 subjects were included in the analysis of accuracy [4].

In the glucose range $<100 \mathrm{mg} / \mathrm{dL}$ in summary $98.8 \%$ of the values and in the range $\geq 100 \mathrm{mg} / \mathrm{dl}$ in summary $98.6 \%$ full filled the quality criteria of the ISO 15197. Important differences between the three tested lots were not shown; detailed data are presented in Tables 1 and
2. The Table 3 demonstrates performance in relation to the minimum accuracy requirements of ISO 15197:2015 where for each of the 3 lots of strips at least $95 \%$ of results must fall within $\pm 15 \mathrm{mg} / \mathrm{dl}$ of the comparison measurement results at blood glucose concentrations $<100$ $\mathrm{mg} / \mathrm{dl}$ and within $\pm 15 \%$ at concentrations $\geq 100 \mathrm{mg} / \mathrm{dl}$. The standard also requires that at least $99 \%$ of individual results fall within consensus error grid zones A and B when clinical accuracy is evaluated with 3 test strip lots [5] (Figure 1).

In conclusion, this study demonstrates that the GL50 evo and GL44 SMBG systems fulfils and exceeds the minimum analytical and clinical accuracy requirement of ISO 15197:2015. There are now differences in the accuracy between the glucose values upper and below $100 \mathrm{mg} / \mathrm{dL}$.

Table 1. System accuracy of Beurer GL50 evo/ GL44 for glucose concentrations $<100$ $\mathrm{mg} / \mathrm{dL}$

\begin{tabular}{|c|c|c|c|}
\hline & \multicolumn{3}{|c|}{ ISO 15197:2015 criteria } \\
\hline Strip lot & within $\pm \mathbf{5 m g} / \mathbf{d L}$ & within $\pm \mathbf{1 0 m g} / \mathbf{d L}$ & within $\pm \mathbf{1 5 m g / d L}$ \\
\hline A $10 / 1$ & $28 / 56(50.00 \%)$ & $53 / 56(94.64 \%)$ & $56 / 56(100 \%)$ \\
\hline A $10 / 3$ & $30 / 56(53.57 \%)$ & $54 / 56(96.43 \%)$ & $55 / 56(98.21 \%)$ \\
\hline A $10 / 4$ & $43 / 56(76.79 \%)$ & $54 / 56(96.43 \%)$ & $55 / 56(98.21 \%)$ \\
\hline $\begin{array}{c}\text { Three lots } \\
\text { in summary }\end{array}$ & $101 / 168(60.12 \%)$ & $161 / 168(95.83 \%)$ & $166 / 168(98.81 \%)$ \\
\hline
\end{tabular}

${ }^{\star}$ Correspondence to: Eckhard Salzsieder, Institute of Diabetes, Gerhardt Katsch, Karlsburg, Germany, E-mail: apuchert@diabetes-karlsburg.de

Key words: conformité européene, ISO, SMBG

Received: August 28, 2019; Accepted: September 25, 2019; Published: September 30, 2019 
Salzsieder E (2019) System accuracy evaluation of the systems for self-monitoring of blood glucose GL50 evo and GL 44 following DIN EN ISO 15197:2015: A comparison of accuracy in glucose concentration ranges $<100 \mathrm{mg} / \mathrm{dL}$ and $\geq 100 \mathrm{mg} / \mathrm{dL}$

Table 2. System accuracy of Beurer GL50 evo/ GL44 for glucose concentrations $\geq 100 \mathrm{mg} / \mathrm{dL}$

\begin{tabular}{|c|c|c|}
\hline & \multicolumn{2}{|c|}{ ISO 15197:2015 criteria } \\
\hline Strip lot & within $\pm \mathbf{5} \%$ & within $\pm \mathbf{1 0} \%$ \\
\hline A $10 / 1$ & $58 / 144(40.28 \%)$ & $112 / 144(77.78 \%)$ \\
\hline A $10 / 3$ & $62 / 144(43.06 \%)$ & $119 / 144(82.64 \%)$ \\
\hline A $10 / 4$ & $62 / 144(43.06 \%)$ & $127 / 144(88.19 \%)$ \\
\hline $\begin{array}{c}\text { Three lots } \\
\text { in summary }\end{array}$ & $182 / 432(42.13 \%)$ & $358 / 432(82.87 \%)$ \\
\hline
\end{tabular}

Table 3. Analytical and Clinical Accuracy of the GL50 evo with 3 Lots of Strips

\begin{tabular}{|c|c|c|c|}
\hline SMBG system & \multicolumn{3}{|c|}{ ISO 15197:2015 criteria within $\pm \mathbf{1 5}$ mg/dl and \pm 15\% } \\
\hline Meter & Strip lot & Individual lots & 3 lots combined \\
\hline GL50 evo/ & A 10/1 & $198 / 200(99.0 \%)$ & $592 / 600(98.7 \%)$ \\
\hline GL44 & A $10 / 3$ & $197 / 200(98.5 \%)$ & \\
\hline & A $10 / 4$ & $197 / 200(98.5 \%)$ & \\
\hline
\end{tabular}

Numbers and percentages of results within system accuracy limits of ISO 15197:2015

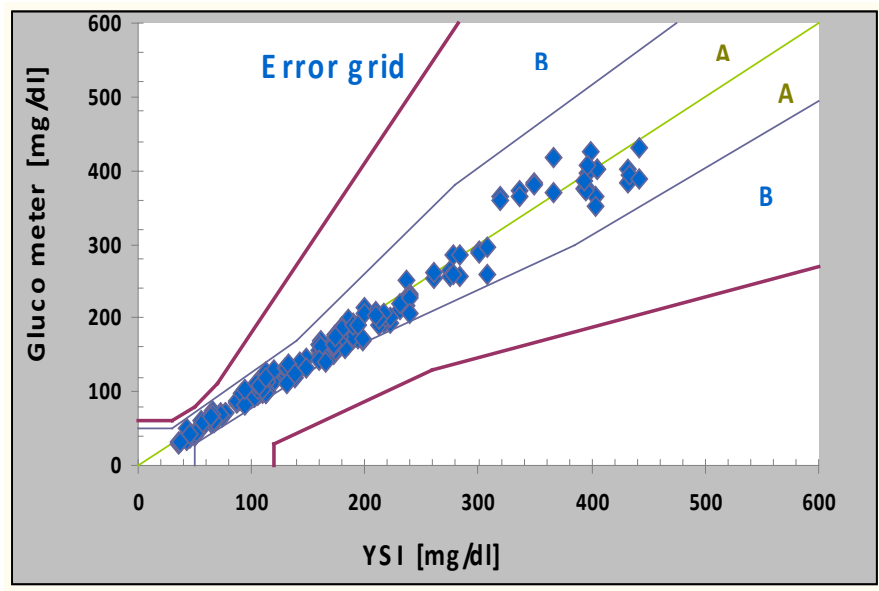

Figure 1. Error Grid Analysis of test Lot 1 (A 10/1), Mean values of GL50 evo vs. mean values of the YSI 2300 Stat Plus

\section{Acknowledgments}

Beurer $\mathrm{GmbH}$ were permitted to review and comment on the manuscript, but final decision on content was retained by the authors.

\section{Declaration of conflicting interests}

The author(s) declared the following potential conflicts of interest with respect to the research, authorship, and/or publication of this article: All authors are employees of the Institute of Diabetes, Karlsburg, Germany, which carries out studies evaluating blood glucose meter systems on behalf of various companies.

\section{Funding}

The author(s) disclosed receipt of the following financial support for the research, authorship, and/or publication of this article: This study was funded by Beurer GmbH Söflinger Str 218, 89077 Ulm Germany.

\section{References}

1. Klonoff DC, Prahalad P (2015) Performance of Cleared Blood Glucose Monitors. $J$ Diabetes Sci Technol 9: 895-910. [Crossref]

2. International Organization for Standardization (2013) In vitro diagnostic test systemsrequirements for blood-glucose monitoring systems for self-testing in managing diabetes mellitus. ISO 15197.

3. Baumstark A, Schmid C, Pleus S, Rittmeyer D, Haug C, et al. (2014) Accuracy assessment of an advanced blood glucose monitoring system for self-testing with three reagent system lots following ISO 15197:2013. J Diabetes Sci Technol 8: 1241-1242. [Crossref]

4. Parkes JL, Slatin SL, Pardo S, Ginsberg BH (2000) A new consensus error grid to evaluate the clinical significance of inaccuracies in the measurement of blood glucose. Diabetes Care 23: 1143-1148.

5. Bland JM, Altman DG (1986) Statistical methods for assessing agreement between two methods of clinical measurement. Lancet 1: 307-310. [Crossref] 